\section{Originalien}

Extrakorporale piezoelektrische Lithotripsie von intra- und extrahepatischen

Gallengangssteinen 1053

\section{Kurze Originalien \& Fallberichte}

Akutes Lungenversagen bei Malaria tropica in der Schwangerschaft 1060

Kritische Extremitätenischämie durch Ergotismus 1067

Mesenterialzyste: seltene Ursache abdomineller Beschwerden 1072
Mediquiz 1077

Aktuelle Diagnostik \& Therapie

Diagnostik und Therapie der Gonorrhoe 1079

Prinzip \& Perspektive

Cushing-Syndrom (Teil II) 1083

Übersichten

Grundlagen und Entwicklungen

in der Homöopathie 1085

\section{Arztrecht in der Praxis}

Zulassung eines teilzeitbeschäftigten Arztes zur Kassenpraxis 1091

Fragen aus der Praxis

Scharlach 1094

Selen 1094

\section{Leser-Zuschriften}

Lambliasis des Magens 1096

\section{Kleine Mitteilungen}

Akute Intoxikationen mit Dibenzepin 1097

Klinische Diagnose der hypertrophischen

Pylorusstenose 1098

Anaphylaxie nach Insektenstich 1099

Hochschulnachrichten 1100 


\section{V1 17 Deutsche Medizinische \\ Wochenschrift}

\section{Schriftleitung}

R. Augustin, Stuttgart

J. F. Riemann, Ludwigshafen

M. Rothmund, Marburg

P. C. Scriba, München

W. Siegenthaler, Zürich

J. R. Siewert, München

A. Sturm, Herne/Bochum

23. Juli 1993

118. Jahrgang, Nr. $29 / 30$
Georg Thieme Verlag

Rüdigerstraße 14

70469 Stuttgart

Postfach 301120

70451 Stuttgart

\section{Inhaltsverzeichnis}

\section{Originalien}

Extrakorporale piezoelektrische Lithotripsie von intraund extrahepatischen Gallengangssteinen-Adamek, H. E., A. Buttmann, C. M. Hartmann, R. Jakobs, J. F. Riemann

Extracorporeal piezoelectric

1053 lithotripsy of intra- and extrahepatic biliary tract stones-Adamek, H. E., A. Buttmann, C. M. Hartmann, R. Jakobs, J. F. Riemann

\section{Kurze Originalien \& Fallberichte}

Akutes Lungenversagen bei Malaria tropica in der Schwangerschaft - Neurath, M., A. Benzing, P. Knolle, H. Grundmann, W. Dippold, K.-H. Meyer zum Büschenfelde

Kritische Extremitätenischämie durch Ergotismus Horstmann, R., H. Daum, M. Heller, A. Schröder

Mesenterialzyste: seltene Ursache abdomineller Beschwerden-Sieg, A., A. Malm, N. Runkel, H. Rausch, C. Herfahrt

\section{Mediquiz}

Acute respiratory failure in falciparum malaria during pregnancy-Neurath, M., A. Benzing, P. Knolle, H. Grundmann, W. Dippold, K.-H. Meyer zum Büschenfelde Critical limb ischaemia caused by ergotism - Horstmann, $R$., H. Daum, M. Heller,

A. Schröder

Mesenteric cyst, a rare cause of abdominal symptoms Sieg, A., A. Malm, N. Runkel, H. Rausch, C. Herfahrt

\section{Aktuelle Diagnostik \& Therapie}

Diagnostik und Therapie der Gonorrhoe-Korting, H. C., M. Kollmann

\section{Prinzip \& Perspektive}

Cushing-Syndrom (Teil II) Ziegler, $R$.

\section{Übersichten}

Grundlagen und Entwicklungen in der Homöopathie Schmidt, J.M.
Diagnosis and treatment of gonorrhoea-Korting, H. C., M. Kollmann

Cushing's syndrome (part II) Ziegler, $R$.

Fundamentals and 1085 developments in homoeopathy -Schmidt, J. M.

\section{Arztrecht in der Praxis}

Zulassung eines teilzeitbeschäftigten Arztes zur Kassenpraxis Rieger, H. $-J$. 
Inhaltsverzeichnis

(Fortsetzung)

\section{Fragen aus der Praxis}

Schmitt, H. J. - Scharlach

1094

Voigtmann, R. - Selen

1094

\section{Leser-Zuschriften}

Grouls, V., A. Schnabel-Lambliasis des Magens

1096

Kleine Mitteilungen

Donatini, B., J. Le Blaye, M. Hall, P. Krupp-

Akute Intoxikationen mit Dibenzepin

Klinische Diagnose der hypertrophischen Pylorusstenose

Anaphylaxie nach Insektenstich

Hochschulnachrichten: Aachen, Bochum, Düsseldorf, Gießen, Heidelberg, Hohenheim, Marburg - Geburtstage - Todesfälle
Alle Manuskripte sind direkt an die Schriftleitung zu richten. Grundsätzlich werden nur solche Arbeiten angenommen, die vorher weder im Inland noch im Ausland veröffentlicht worden sind. Die Arbeiten dürfen auch nicht gleichzeitig anderen Zeitschriften zum Abdruck angeboten werden. - Auch angeforderte Beiträge werden nicht in jedem Fall zur Publikation übernommen; die endgültige Entscheidung ist erst nach zusätzlicher Prüfung durch Fachgutachter möglich. Die Schriftleitung behält sich eine redaktionelle Überarbeitung vor

Für Angaben zu Dosierung und Applikationsform von Medikamenten kann vom Verlag keine Gewähr übernommen werden. Derartige Angaben müssen vom Anwender auf ihre Richtigkeit überprüft werden. Mit der Annahme und Veröffentlichung des Manuskriptes erwirbt der Verlag für die Dauer der gesetzlichen Schutzfrist ( $\$ 64 \mathrm{UrhG}$ ) die ausschließliche Befugnis zur Wahrnehmung der Verwertungsrechte im Sinne der $\S \S 15 \mathrm{ff}$. des Urheberrechtsgesetzes, insbesondere auch das Recht der Übersetzung, der Vervielfältigung durch Photokopie oder ähnliche Verfahren und der EDV-mäßigen Verwertung. Photokopien für den persönlichen und sonstigen eigenen Gebrauch dürfen nur von einzelnen Beiträgen oder Teilen daraus als Einzelkopien hergestellt werden. Die Zeitschrift erscheint wöchentlich (Doppelhefte im Januar, Juli, August und Dezember). - (c) Georg Thieme Verlag Stuttgart · New York 1993. - Printed in Germany.

For users in the USA

Authorization to photocopy items for internal or personal use, or the internal or personal use of specific clients, is granted by Georg Thieme Verlag Stuttgart - New York for libraries and other users registered with the Copyright Clearance Center (CCC) Transactional Reporting Service, provided that the base fee of $\$ 02.00$ per copy of each article is paid directly to CCC, 27 Congress St., Salem, MA 01970. 0012-0472/93 \$02.00. For reprint information in the United States, contact: International Reprint Corporation, 968 Admiral Callaghan Lane \# 268, P.0.Box 12004 , Vallejo, CA 94590 U.S.A., Telephone (707) 553-9239, Fax (707) 552-9524 This journal is regularly listed in Current Contents (CM/LS), Science Citation Index and Index Medicus.

ISSN 00 12-0472

\begin{tabular}{llll}
\hline Bezugspreise $^{\star}$ & $\begin{array}{l}\text { Abonnements- } \\
\text { preis }\end{array}$ & $\begin{array}{l}\text { Versand- } \\
\text { kosten }\end{array}$ & Gesamt \\
\hline $\begin{array}{l}\text { Jahresbezugspreis } \\
\text { Inland }\end{array}$ & DM 258,- & DM 39,- & DM 297,-- \\
Ausland & DM 258,- & $\begin{array}{c}\text { DM 78,- } \\
\text { DM 336,- }\end{array}$ \\
$\begin{array}{l}\text { Vorzugspreis für Ärzte in der Weiterbildung zum Gebietsarzt } \\
\text { (nur im ersten Jahr des Abonnements) }\end{array}$ & DM 39,- & DM 213,- \\
Inland & DM 174,- & DM 78,- & DM 252,- \\
Ausland & DM 174,- & DM & \\
Vorzugspreis für Studenten und Ärzte im Praktikum & \\
Inland & DM 88,- & DM 39,- & DM 127,- \\
Ausland & DM 88,- & DM 78,- & DM 166,-- \\
\hline
\end{tabular}

Einzelheft DM 9,-, zuzüglich Versandkosten ab Verlagsort. Alle Preise sind unverbindlich empfohlene Preise. Preisänderungen vorbehalten. Luftpostgebühren: Europa DM 118,-; USA/Kanada DM 160,-; übrige Länder DM 237,50. Falls gewünscht, bitte bei Bestellung angeben. Die Bezugsdauer verlängert sich jeweils um $1 \mathrm{Jahr}$, wenn nicht eine Abbestellung bis zum 30 . September vorliegt. * Unverbindlich empfohlener Preis

Verantwortlich für die Schriftleitung: Dr. med. habil. R. Augustin, Rüdigerstraße 14, 70469 Stuttgart, Telefon 0711/8931-231. Verantwortlich für den Anzeigenteil: pharmedia Anzeigen- und Verlagsservice GmbH, Postfach 301120, 70451 Stuttgart, Rüdigerstraße 14, 70469 Stuttgart, Telefon 0711/8931-0, Telex 0723644 p med d. Druck: Druckhaus Götz GmbH, Schwieberdinger Straße 111-113, 71636 Ludwigsburg. Telefon 07141/41058, Telefax 07141/41519. Verlag: Georg Thieme Verlag, Postfach 301120, 70451 Stuttgart, Rüdigerstraße 14, 70469 Stuttgart, Telefon-Sammel-Nr. $0711 / 8931-0$, Telefax $0711 / 8931-298$, Telex 7252275 gtv d, Telegrammadresse: Thiemebuch Stuttgart. Mitglied der Informationsgemeinschaft zur Feststellung der Verbreitung von Werbeträgern. 


\title{
Grundlagen und Entwicklungen in der Homöopathie
}

\author{
J. M. Schmidt \\ Institut für Geschichte der Medizin der Universität München
}

Nicht erst seit Inkrafttreten der neuen Studienordnung für Medizin - seit 1993 ist die Homöopathie Bestandteil des Gegenstandskatalogs für den Zweiten Abschnitt der Ärztlichen Prüfung (28) und damit Lehrfach an den Medizinischen Fakultäten deutscher Universitäten - werden von künftigen Ärzten auch Grundkenntnisse in sogenannten alternativen Therapieverfahren verlangt, wozu auch die Homöopathie gerechnet wird. Schon seit längerem sehen sich niedergelassene Mediziner einer zunehmend in diesem Sinne veränderten Erwartungshaltung ihrer Patienten gegenüber. Dementsprechend steil sind in den letzten Jahren auch die Teilnehmerzahlen an diesbezüglichen ärztlichen Weiterbildungs- und Fortbildungskursen angestiegen.

Angesichts des allgemeinen Trends zu einer vermehrten Einbeziehung bislang als »parauniversitär « eingestufter Heilmethoden in die reguläre medizinische Ausbildung und Praxis besteht nun ein relativer Nachholbedarf an wissenschaftlich fundierten Arbeiten etwa zu den Prinzipien und Problemen der Homöopathie - erhob sich doch die verfügbare homöopathische Literatur, die aus mehreren tausend Monographien und mehreren hundert Zeitschriftentiteln besteht, bislang eher selten über den Rang unkritischer Apologien bzw. inkompetenter Schmähschriften von seiten ihrer Gegner.

Neben grundsätzlichen Hindernissen eines ersten Zugangs zur Homöopathie (zum Beispiel ihre eigene, historisch bedingte Terminologie und ihr besonderer, philosophisch erschließbarer Ansatz) (22) liegt eine der Hauptschwierigkeiten des Verständnisses ihrer eigentlichen Grundlagen darin, daß die mannigfachen späteren Modifikationen und Umdeutungen der ursprünglichen Lehre in der bisherigen Literatur meist nicht als solche erkannt bzw. systematisch dargestellt wurden.

Dtsch. med. Wschr. 118 (1993), 1085-1090

(C) Georg Thieme Verlag Stuttgart · New York

\section{Differenzierung der Prinzipien der Homöopathie}

Um so wichtiger wäre es daher, bereits zu Beginn jeder eingehenderen Beschäftigung mit der Homöopathie differenzieren zu lernen zwischen

- den Prinzipien, die konstitutiv für die Homöopathie sind - Prinzipien also, mit welchen sie als eigenständige Arzneitherapie steht und fällt,

- den Prinzipien, die von ihrem Begründer erst in vorgerücktem Alter, lange nach der eigentlichen Grundlegung der Homöopathie, ergänzt wurden Prinzipien also, die sich bewähren oder nicht bewähren können, ohne daß damit die Homöopathie als solche bewiesen oder widerlegt wäre, und

- den Prinzipien, die erst von späteren Homöopathen als »Weiterentwicklungen« der Lehre eingeführt wurden - Prinzipien also, die mit Vorbehalt $\mathrm{zu}$ betrachten und nicht zur Homöopathie im engeren Sinne zu rechnen sind.

\section{Samuel Hahnemann (1755-1843)}

Begründet wurde die Homöopathie im Jahr 1796 (5), zu einer Zeit, als in der Medizin ein genereller Pluralismus von Systemen vorherrschte und vor allem die Therapie allgemein anerkannter Grundlagen entbehrte.

Eine sichere, rationale Grundlage für die Arzneitherapie zu schaffen, war aber das Lebensziel des 1779 promovierten Arztes Samuel Hahnemann (1755-1843), eines aufklärerischen Geistes, der sich in relativ jungen Jahren schon bedeutende Verdienste um die Wissenschaft erworben hatte (24). Bereits kurz nach seiner ersten Niederlassung hatte Hahnemann - aus Verzweiflung an der desolaten Lage der damaligen Medizin - seine Praxis fast ganz aufgegeben und hauptsächlich von der Publikation seiner chemischen, pharmazeutischen, psychiatrischen, hygienischen und gerichtsmedizinischen Forschungen sowie von Übersetzungen medizinischer Standardwerke gelebt. Erst nachdem er - als Ver- 
such eines Auswegs aus dieser mißlichen therapeutischen Situation (23) - die Homöopathie begründet hatte, wie sie ab 1810 in seinem »Organon der rationellen Heilkunde« vorlag (6), nahm er seine Praxis wieder voll auf, lehrte ab 1812 als Privatdozent an der Universität Leipzig und praktizierte nunmehr bis zu seinem Tod im Alter von 88 Jahren.

\section{Die Grundprinzipien der Homöopathie}

\section{Die Verwendung von Einzelmitteln}

Bei seiner Kritik der damaligen Heilkunde galt Hahnemanns Hauptaugenmerk der Arzneitherapie. Hier verurteilte er aufs Schärfste die gewöhnliche Praxis, Gemische von verschiedenen Arzneisubstanzen zu verabreichen, da man auf diese Weise weder deren Gesamtwirkung voraussehen, noch etwas über die Wirkungsweise der einzelnen Bestandteile lernen könne. Mit Hahnemanns Grundsatz, selbst fortan nur noch Einzelmittel zu verwenden, war das erste seiner Prinzipien zur Begründung einer rationalen Arzneitherapie festgelegt.

\section{Die Arzneimittelprüfung an Gesunden}

Wollte man etwas über die Wirkungen von Medikamenten erfahren, war man auf die damaligen Arzneimittellehren angewiesen. Diese beruhten meist entweder auf der Überlieferung empirischer Zufallsbefunde durch Laien oder aber auf Spekulationen über vermeintliche »Tugenden« von Arzneistoffen. Da beide Quellen für Hahnemann keinen wissenschaftlichen Wert hatten, forderte er statt dessen für jede Arzneisubstanz deren experimentelle Überprüfung am Menschen - und zwar am gesunden Menschen, damit nicht etwa Krankheitssymptome fälschlich als Wirkungen der Arzneien interpretiert würden. Auf diese Weise gelangte Hahnemann schließlich zu einer »Reinen Arzneimittellehre«, die weder Gerüchte noch Spekulationen, sondern ausschließlich mit den fünf Sinnen wahrgenommene Symptome beinhalten sollte. Mit diesem neuen Ansatz einer experimentellen Pharmakologie stand das zweite Prinzip von Hahnemanns rationaler Arzneitherapie fest: die Arzneimittelprüfung an gesunden Menschen.

\section{Das Ähnlichkeitsprinzip}

Das eigentlich therapeutische Prinzip, das seiner Lehre schließlich den Namen gab, fand Hahnemann aufgrund mehrerer therapeutischer Beobachtungen. Zum einen hatte man bemerkt nicht zuletzt im Zusammenhang mit der gleichzeitigen Entdeckung der Vakzination durch Edward Jenner (1749-1823) (15) -, daß eine Krankheit manchmal durch Hinzutreten einer anderen, ähnlichen ausgelöscht und damit »geheilt « werden konnte (wie etwa die Kuhpocken durch Menschenpocken). Zum anderen hatte Hahnemann selbst beobachtet, daß zum Beispiel Chinarinde, die das damalige Spezifikum bei Wechselfieber war, bei einer Arzneimittelprüfung am Gesunden eben solche Symptome erzeugte, wie sie ihm selbst vom Wechselfieber her bereits bekannt waren. Hahnemanns Überlegung war nun folgende: Wenn eine natürliche Krankheit durch eine andere, ihr ähnliche natürliche Krankheit ausgelöscht werden konnte, so müßte dies auch mit Hilfe einer bewußt erzeugten, künstlichen Arzneikrankheit möglich sein. Nach mehrjährigen Versuchen fühlte sich Hahnemann 1796 schließlich berechtigt, als neues Therapieprinzip folgendes aufzustellen: Behandle Krankheiten mit einem Mittel, das bei der Arzneimittelprüfung an Gesunden solche Symptome hervorbringen kann, die denen des Kranken möglichst ähnlich sind (5). Mit diesem Grundsatz stand das dritte Prinzip von Hahnemanns rationaler Arzneitherapie fest: das Simile-Prinzip (similia similibus curentur - Behandle Ähnliches mit Ähnlichem).

Wie der Konjunktiv in »curentur" zeigt, hatte Hahnemann im Ähnlichkeitsprinzip ursprünglich lediglich eine therapeutische Maxime gesehen, und noch nicht - wie erst in späteren Jahren - ein vermeintliches »Naturgesetz«.

\section{Die Verwendung kleinster Arzneigaben}

Wie vorauszusehen, traten bei der Verabreichung solcher Substanzen, die von sich aus ähnliche Symptome erzeugen konnten, wie sie bei den einzelnen Patienten bereits vorlagen, erhebliche Verschlimmerungen ihres Zustandes auf. Dies war nun der Grund für Hahnemann, die Dosis seiner Arzneimittel mehr und mehr zu verkleinern. Durch schrittweises Verdünnen derselben (meist im Verhältnis $1: 100$ ) versuchte er, die Stärke seiner Arzneigaben so weit abzuschwächen, daß einerseits zwar ihre Wirkung auf den Organismus noch ausreichend war, andererseits aber die zu erwartende sogenannte Erstverschlimmerung möglichst unauffällig verlaufen sollte. Damit war Hahnemann beim vierten Prinzip seiner rationalen Arzneitherapie angelangt: der Verwendung kleinster Arzneigaben.

Hiermit war - von ihren Prinzipien her - die Begründung der »allgemeinen Homöopathie« abgeschlossen. Sowohl historisch als auch logisch bauen diese Prinzipien aufeinander auf/und alle vier sind konstitutiv für Hahnemanns ursprünglichen Entwurf einer rationalen Arzneitherapie. Was von seiten Hahnemanns und seiner Schüler nun noch folgte, war zum einen die genaue Prüfung von zahlreichen Arzneistoffen an gesunden Probanden, zum anderen die Ausarbeitung einer verfeinerten Methodik der Anwendung der genannten Prinzipien im konkreten Einzelfall. 


\section{Von Hahnemann später ergänzte Prinzipien}

Obwohl es Hahnemanns ausdrückliche Absicht war, eine von Vermutungen und Spekulationen freie rationale und empirisch nachprüfbare Arzneitherapie zu schaffen, und er bloße Theorien und Hypothesen aus der Heilkunde grundsätzlich ausschließen wollte, finden sich eben solche nichtsdestotrotz in seinen eigenen späteren Schriften. Wenn er auch anfangs den zunehmenden Einfluß der Naturphilosophie in der Medizin des beginnenden 19. Jahrhunderts noch kritisiert hatte, so war er doch in den letzten beiden Jahrzehnten seines Lebens weniger von der schon verblaßten Aufklärung als mehr und mehr von der Zeit der deutschen Romantik bestimmt $(19,25)$.

Aus dieser späten Zeit stammen einige Spekulationen Hahnemanns, die er selbst größtenteils zwar als "nur wahrscheinliche Annahmen« bezeichnete, die aber in der Rezeption seiner Werke oft fälschlicherweise für die eigentlichen Grundlagen der Homöopathie gehalten und entsprechend überbewertet wurden.

\section{Die »Lebenskraft« und ihre »Verstimmung«}

Einer der Begriffe, die bei der eigentlichen Begründung der Homöopathie praktisch keine Rolle spielten und erst in den späteren Schriften Hahnemanns zunehmende Bedeutung erlangten, ist der der »Lebenskraft«. Erst im letzten Drittel seiner rund 60jährigen Schaffensperiode versuchte Hahnemann vermehrt, Krankheit als Produkt einer »verstimmten «, geistartig vorgestellten »Lebenskraft « zu erklären und Heilung als Resultat einer Einwirkung der Arzneikraft direkt auf diese. Gemäß einer Erläuterung, die Hahnemann fünf Jahre vor seinem Tod gab, werde der »Lebenskraft« durch die Wirkung des homöopathischen Arzneimittels sozusagen ein ähnlicher »Krankheitsfeind « »vergrößert vorgehalten«, wodurch jene letztlich veranlaßt werde, ihre eigene Energie so weit zu erhöhen, daß sie den Organismus schließlich wieder ganz beherrschen könne (11).

Zu dieser Art von »Erklärungs-Versuchen « der homöopathischen Arzneiwirkung ist allerdings zu bemerken, daß sich Hahnemann ihres spekulativen Charakters durchaus bewußt war und er ausdrücklich nicht die Absicht hatte, damit etwa die Grundlagen der Homöopathie zu beweisen.

\section{Das »Potenzieren«von Arzneimitteln}

Eine weitere spätere Vorstellung Hahnemanns, die dieser erst lange nach der eigentlichen Begründung der Homöopathie erstmals geäußert hat, die dennoch aber meist gerade als das Wesent- lichste der Homöopathie angesehen wird, ist das Konzept des »Potenzierens« bzw. »Dynamisierens« von Arzneimitteln.

Wie bereits erwähnt, hatte Hahnemann zunächst versucht, durch schrittweises Verdünnen seiner Arzneigaben deren Wirkung so weit abzuschwächen, daß die zu erwartende Erstverschlimmerung möglichst mild verlaufen sollte. $\mathrm{Zu}$ seiner Überraschung bemerkte er bald, da $ß$ - so weit er dieses schrittweise Verdünnen auch trieb - er keine Grenze finden konnte, ab der die Verdünnung eines homöopathisch passenden Mittels keine Wirkung mehr zu zeigen schien. Obwohl ihm die Macht der Suggestion und auch die Placebo-Wirkung von reinem Milchzucker nachweislich bekannt waren, nahm er dieses Phänomen ohne derartige Interpretationen zur Kenntnis und bemerkte dazu nur, daß er »es selbst nicht begreife« (10). - Fast sein ganzes Leben lang behandelte Hahnemann allerdings mit Verdünnungsgraden, die von Urtinkturen bis maximal zur 30. Stufe reichten.

Erst 1821, im Rahmen der sich verschärfenden Auseinandersetzungen mit den Leipziger Ärzten und Apothekern, entwickelte Hahnemann schließlich das Konzept, daß das mit Reiben und Schütteln verbundene Verdünnen einer Arzneimittel-Auflösung nicht nur deren Entstofflichung, sondern auch deren »Vergeistigung « bewirken sollte (7). Durch den »Potenzierungs«-Vorgang, wie er das Verdünnen und Verschütteln ab 1827 nannte (8), würden die sonst schlummernden Arzneikräfte einer Substanz überhaupt erst freigesetzt und die Auflösung mit jeder »Potenzierung « stärker wirksam werden. - Die Folge dieser Theorie war zum einen der nunmehr einsetzende Trend von seiten der orthodoxen Anhänger Hahnemanns zur Herstellung und Verwendung von »Hochpotenzen«, zum anderen die Abspaltung der sogenannten naturwissenschaftlichkritischen Homöopathen, die sich nicht vorstellen konnten, daß mit zunehmender Verdünnung und Verschüttelung einer Substanz deren Wirksamkeit zunehmen sollte.

Bis zum heutigen Tag ist diese Annahme Hahnemanns und seiner Nachfolger der eigentliche Stein des Anstoßes der Homöopathie geblieben. Nach wie vor wird mit dem Hinweis auf die Loschmidtsche Zahl, nach der sich spätestens in einer C12-Auflösung (entsprechend einer Verdünnung von $10^{-24}$ ) kein Molekül der Ausgangssubstanz mehr befinden könne, versucht, die Homöopathie als Ganzes ad absurdum zu führen. - Nicht berücksichtigt wird bei dieser Schlußfolgerung, daß es sich bei der Frage der Wirksamkeit von »Hochpotenzen« lediglich um ein innerhomöopathisches Problem handelt, von dem strenggenommen kein einziges der vier genannten Grundprinzipien der Homöopathie betroffen wäre. 
Derzeit liegen etwas über hundert Studien vor (in vitro sowie an Pflanzen, Tieren und Menschen; 21, 24), nach denen selbst ultra-molekulare Verdünnungen und Verschüttelungen in der Lage zu sein scheinen, biologische Effekte auszulösen $(2,20)$. Einer kritischen Meta-Analyse dieser Arbeiten zufolge sollen »die bisherigen Ergebnisse zu weiteren, gut durchgeführten Studien berechtigen« (18).

\section{Die »Psora« als Ursache chronischer Krankheiten}

Auch die von Hahnemann mit 73 Jahren aufgestellte »Psora-Theorie« hatte schwerwiegende Folgen für die weitere Rezeption der Homöopathie. Ausgehend von dem Eingeständnis, daß die »allgemeine Homöopathie« bei chronischen Krankheiten oft keine Heilung brachte, entwickelte Hahnemann eine Theorie, mit deren Hilfe letztlich auch diese Lücke seines Heilsystems geschlossen werden sollte. Nach dieser Hypothese sollten alle nicht-venerischen chronischen Krankheiten auf einer früheren Ansteckung mit Krätze beruhen. Obwohl Hahnemann 36 Jahre zuvor als Erreger der Krätze selbst bereits eindeutig die Krätz-Milbe beschrieben hatte $(3,4)$, erwähnte er diese hier mit keinem Wort. Vielmehr stellte er sich Wesen und Verlauf der »inneren Krätzkrankheit« oder »Psora« jetzt in Analogie zur Syphilis vor: zunächst eine Ansteckung über die Haut, während einer Inkubationszeit dann Ausbreitung der Krankheit im ganzen Organismus, danach Bildung des lokalen Hautausschlags und bei bloß örtlicher Behandlung desselben schließlich Aktivierung der im Inneren des Organismus schlummernden Gesamt-Krankheit (9).

Bei allen Unzulänglichkeiten dieses Entwurfs aus heutiger Sicht sollte - ohne hier auf Einzelheiten eingehen zu können - zumindest zweierlei festgehalten werden:

- Erstens bezeichnete Hahnemann diesen seinen Entwurf selbst als »Theorie«, der lediglich eine bestimmte »Wahrscheinlichkeit« zukomme und nach der man nur so lange verfahren solle, wie es noch keine wahrscheinlichere andere Theorie dazu gebe.

- Zweitens ließ auch die »Psora-Theorie« die Grundprinzipien der Homöopathie unangetastet. Praktisch-therapeutisch änderte sich lediglich der Umfang der zu erhebenden biographischen Anamnese sowie die Einbeziehung auch nur potentiell vorhandener »psorischer « Symptome in die Arzneimittelwahl.

\section{Spätere »Weiterentwicklungen« der Homöopathie}

Neben diesen von Hahnemann nur in seinen späten Schriften geäußerten Prinzipien finden sich in der homöopathischen Literatur aber auch solche, die erst nach Hahnemanns Tod von dessen Nachfolgern als »Weiterentwicklungen« der Homöopathie eingeführt wurden. Auch diese sollten von deren eigentlichen Grundlagen unterschieden werden.

\section{Die Heringsche Regel}

Eine dieser späteren Ergänzungen der homöopathischen Lehre beruhte auf der Beobachtung, daß während der Arzneitherapie von Krankheiten die einzelnen Symptome der Patienten manchmal in einer bestimmten Reihenfolge verschwanden. Nachdem Constantin Hering (1800-1880) verschiedene Überlegungen zu diesem Thema publiziert hatte $(13,14)$, wurde hieraus später folgender Satz extrahiert: »Die Symptome einer Krankheit sollten von oben nach unten, von innen nach außen und in der umgekehrten Reihenfolge ihres Auftretens verschwinden«. Obwohl Hering dies so gar nicht formuliert hatte, wurde dieser Satz dennoch fortan als »Heringsche Regel« tradiert und nicht selten $\mathrm{zu}$ den wichtigsten Grundlagen der Homöopathie gerechnet. Manche Autoren sprachen sogar vom »Heringschen Gesetz«, als gäbe es davon keine Ausnahmen $(17,26)$.

Strenggenommen handelt es sich hierbei um eine Verallgemeinerung einzelner Beobachtungen, die in dieser strikten Form möglicherweise nicht auf die Mehrzahl homöopathischer Heilungsverläufe zutreffen dürfte.

\section{Die »Ultra-Hochpotenzen«}

Eine weitere vermeintliche »Weiterentwicklung« war - im Bereich der homöopathischen Posologie (Gabenlehre) - die Einführung von »Ultra-Hochpotenzen«.

Während Hahnemann seine Auflösungen stets im sogenannten Mehrglasverfahren herstellte (er verwendete pro $1: 100$-Verdünnungsschritt jeweils ein neues Fläschchen), wurde noch zu seinen Lebzeiten von Korsakoff bereits mit der Produktion von sogenannten Einglas-»Potenzen « begonnen, zumal sich dieses Verfahren als wesentlich einfacher und billiger erwies (hier wurden sämtliche $1: 100$-Verdünnungsschritte in ein und demselben Fläschchen vorgenommen). - Völlig neue Dimensionen bekam die »Potenzierung « von Medikamenten jedoch, als vor allem in den USA - etwa von Fincke, Kent, Skinner und H. C. Allen - Maschinen konstruiert wurden, welche die kontinuierliche Herstellung selbst höchster »Potenzstufen« erlaubten. 
Während aber zum Beispiel die Maschine von Skinner zumindest noch das Korsakoffsche Einglas-»Potenzierungsverfahren« imitierte, beruhte etwa beim Fluxionspotentiometer von Allen oder Fincke der vermeintliche »Potenzierungsvorgang« allein auf einer in der Verdünnungsflüssigkeit erzeugten kontinuierlichen Wirbelbildung, die durch einen mit hohem Druck durch eine gewundene Röhre und enge Düsen gepreßten Wasserstrahl bewirkt wurde. So kamen 10000 ste, 100000 ste, Millionste und höhere »Potenzen« auf den Markt und wurden als homöopathische Mittel verordnet, obwohl deren Herstellungsweise mit den ursprünglichen Vorschriften Hahnemanns kaum noch etwas gemeinsam hatte. Erst seit der jüngsten Vergangenheit bemüht man sich auch in den Vereinigten Staaten wieder vermehrt um entweder streng nach Hahnemann oder streng nach Korsakoff hergestellte »Arzneipotenzen«.

\section{Die Kentschen Arzneimittelbilder}

Eine der Hauptschwierigkeiten sowohl für Dozenten als auch Studenten der Homöopathie war immer wieder die Beherrschung der ebenso umfang- wie facettenreichen homöopathischen Arzneimittellehre. James Tyler Kent (1849-1916), selbst langjähriger Professor für Materia Medica in Chicago, hatte nun eine besondere Begabung, einerseits durch ein plastisches Ausmalen bestimmter Arzneimittelsymptome, andererseits aber auch durch markante Ergänzungen aus seiner eigenen klinischen Erfahrung, vielen der Arzneimittel eine Art Persönlichkeit zu verleihen bzw. ihre Charakteristika sozusagen $\mathrm{zu}$ dramatisieren. Aus den trockenen Listen zusammenhangloser Prüfungssymptome entstanden auf diese Weise die anschaulichen »Arzneimittelbilder«, die bei Studenten aufgrund ihrer leichteren Einprägsamkeit naturgemäß großen Anklang fanden (16). Diese von Kent ausgelöste Tendenz des Malens von »Arzneimittelbildern « wirkt bis heute fort, etwa in Form von George Vithoulkas' »Essenzen« (27) oder Catherine Coulters »Portraits« der homöopathischen Materia Medica (1).

Im Gegensatz zu den ursprünglichen Symptomenverzeichnissen, die entweder auf Arzneimittelprüfungen oder auf klinisch verifizierten Heilwirkungen, jedoch stets auf nachprüfbaren Quellen beruhten, hat diese neuere Art von Arzneimittellehren allerdings den Schönheitsfehler, daß hier im Einzelfall zwischen Dichtung und Wahrheit nicht immer sicher zu unterscheiden ist.

Neben diesen drei wichtigsten $»$ Weiterentwicklungen« der Homöopathie gab es im Laufe ihrer langen Geschichte noch eine Unzahl anderer vermeintlicher Neuerungen oder Abzweigungen, die hier nicht im einzelnen dargestellt werden können. Stellvertretend seien nur genannt: die Isopathie von
Lux, die »Biochemie« nach Schüssler, die Konstitutionslehre von v. Grauvogel, der Tuberkulinismus von Vannier, die Umdeutung der Miasmenlehre durch Ortega oder durch Masi-Elizalde, die Einbeziehung der Psychologie, Astrologie und Mythologie, die Zuordnung homöopathischer Mittel zu bestimmten Akupunkturpunkten usw.

All diese Abzweigungen der Homöopathie im weitesten Sinne ließen sich wesentlich leichter beurteilen und voneinander abgrenzen, wenn man sie an Hahnemanns ursprünglich streng rationalem Ansatz messen und den eigentlichen Grundprinzipien der Homöopathie gegenüberstellen würde.

Sollten sich darüber hinaus Apologeten und Kritiker der Homöopathie zunächst einmal über den Stellenwert der jeweils diskutierten Prinzipien innerhalb dieses Therapiesystems verständigen, müßte es möglich sein, künftig zu einer nüchterneren Betrachtung der Homöopathie als bisher zu gelangen. Indem sie allerdings teilweise von Prinzipien ausgeht, die heute nicht jedermann unmittelbar plausibel erscheinen, und ihre individuelle Anwendung prinzipiell nicht nach Indikationen oder ähnlichem standardisierbar ist, wird - abgesehen von der allgemeinen Vermittlung ihrer Grundlagen und Methodik - ihre tatsächliche Beherrschung und Ausübung wohl kaum von jedem Arzt zu verlangen sein. 


\section{Literatur}

1 Coulter, C. R.: Portraits of Homoeopathic Medicines (Homoeopathic Educational Services: Berkeley 1986-1989).

2 Gibson, R. G., S. L. M. Gibson, A. D. MacNeill, W. W. Buchanan: Homoeopathic therapy in rheumatoid arthritis. Evaluation by double-blind clinical therapeutic trial. Brit. J. clin. Pharmacol. 9 (1980), 453-459.

3 Hahnemann, S.: Zusatz. Anzeiger (1792), 2,23/24, $190 f$.

4 Hahnemann, S.: Ueber den Ansprung (crusta lactea). Med. Bibl. 3 (1795), 4, 701-705.

5 Hahnemann, S.: Versuch über ein neues Prinzip zur Auffindung der Heilkräfte der Arzneisubstanzen, nebst einigen Blikken auf die bisherigen. Journal der practischen Arzneykunde und Wundarzneykunst 2 (1796), 3,391-439 u. 4,465-561.

6 Hahnemann, S.: Organon der rationellen Heilkunde (Arnold: Dresden 1810).

7 Hahnemann, S.: Reine Arzneimittellehre. 6. Teil (Arnold: Dresden 1821), V-XVI.

8 Hahnemann, S.: Reine Arzneimittellehre. 2. Aufl. 6. Teil (Arnold: Dresden-Leipzig 1827), XI.

9 Hahnemann, S.: Die chronischen Krankheiten, ihre eigenthümliche Natur und homöopathische Hnilung. 1. Teil (Arnold: Dresden-Leipzig 1828).

10 Hahnemann, S.: Die chronischen Krankheiten, ihre eigenthümliche Natur und homöopathische Heilung. 2. Aufl., 1. Teil (Arnold: Dresden-Leipzig 1835), 154.

11 Hahnemann, S.: Die chronischen Krankheiten, ihre eigenthümliche Natur und homöopathische Heilung. 2. Aufl., 4. Teil (Schaub: Düsseldorf 1838), VI-VIII.

12 Hahnemann, S.: Organon der Heilkunst. Textkritische Ausgabe der 6. Aufl. (1842). Bearbeitet und herausgegeben von J. M. Schmidt (Haug: Heidelberg 1992).

13 Hering, C.: Preface. In Hahnemann, S.: The Chronic Diseases. Their Specific Nature and Homoeopathic Treatment. Translated by C. J. Hempel (Radde: New York 1845), 4-10.

14 Hering, C.: Hahnemann's three rules concerning the rank of symptoms. Hahnem. Monthly 1 (1865), 5-12.

15 Jenner, E.: An Inquiry into the Causes and Effects of the Variolae Vaccinae ... (Low: London 1798).

16 Kent, J. T.: Lectures on Homoeopathic Materia Medica (Boericke \& Tafel: Philadelphia 1905)

17 Kent, J. T.: Correspondence of organs, and the direction of cure. Trans. Soc. Homoeopath. 1 (1911), 31-33.

18 Kleijnen, J., P. Knipschild, G. t. Riet: Clinical trials of homoeopathy. Brit. med. J. 302 (1991), 316-323.

19 Leibbrand, W.: Die spekulative Medizin der Romantik (Claassen: Hamburg 1956), 201-225.

20 Reilly, D. T., M. A. Taylor, C. McSharry, T. Aitchison: Is homoeopathy a placebo response? Controlled trial of homoeopathic potency, with pollen in hayfever as model. Lancet 1986/II, $881-886$

21 Righetti, M.: Forschung in der Homöopathie (Burgdorf: Göttingen 1988).

22 Schmidt, J. M.: Die philosophischen Vorstellungen Samuel Hahnemanns bei der Begründung der Homöopathie (bis zum Organon der rationellen Heilkunde, 1810) (Sonntag: München 1990).

23 Schmidt, J. M.: Der Simile-Weg als deuteros plous in der Arzneitherapie - Konzeption und Rezeption. Docum. homoeopath. 12 (1992), 51-59.

24 Schmidt, J. M.: Alternative oder Anachronismus? Die Behandlung chronischer Krankheiten mittels Homöopathie. In Hammer, C., V. Schubert (Hrsg.): Chronische Krankheiten und ihre Bewältigung (R. S. Schulz: Percha 1993), 202-246.

25 Tischner, R.: Hahnemann und die Romantik. Allg. homöopath. Ztg 201 (1956), 313-318.

26 Vithoulkas, G.: The Science of Homeopathy (Grove: New York 1980), 231, 240

27 Vithoulkas, G.: The Essence of Materia Medica (Jain: New Delhi 1988).

28 Institut für medizinische und pharmazeutische Prüfungsfragen: Gegenstände, auf die sich der schriftliche Teil des zweiten Abschnitts der Ärztlichen Prüfung beziehen kann (= GK 3). (IMPP: Mainz 1993), 330f.: Kap. »Homöopathie«.
Dr. med. Dr. phil. J. M. Schmidt Institut für Geschichte der Medizin der Universität Lessingstr. 2 80336 München 